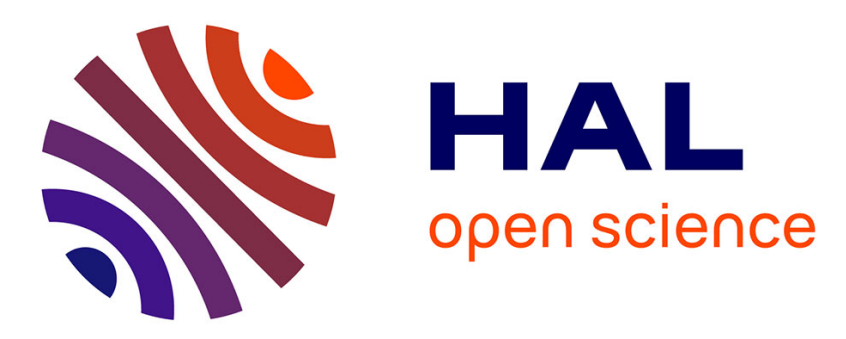

\title{
Tracking points on deformable objects using curvature information
}

Isaac Cohen, Nicholas Ayache, P. Sulger

\section{To cite this version:}

Isaac Cohen, Nicholas Ayache, P. Sulger. Tracking points on deformable objects using curvature information. [Research Report] RR-1595, INRIA. 1992. inria-00074965

\section{HAL Id: inria-00074965 \\ https://hal.inria.fr/inria-00074965}

Submitted on 24 May 2006

HAL is a multi-disciplinary open access archive for the deposit and dissemination of scientific research documents, whether they are published or not. The documents may come from teaching and research institutions in France or abroad, or from public or private research centers.
L'archive ouverte pluridisciplinaire HAL, est destinée au dépôt et à la diffusion de documents scientifiques de niveau recherche, publiés ou non, émanant des établissements d'enseignement et de recherche français ou étrangers, des laboratoires publics ou privés. 


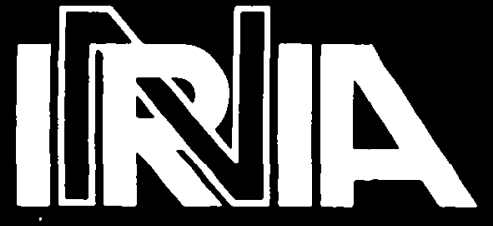

UNITÉ DE RECHERCHE INRIA-ROCQUENCOURT

\section{Rapports de Recherche}

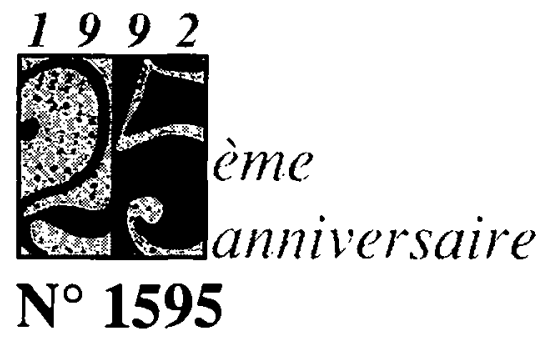

\section{Programme 4}

Robotique, Image et Vision

TRACKING POINTS ON DEFORMABLE OBJECTS USING CURVATURE INFORMATION
Institut National de Recherche en Informatique et en Automatique

Domaine de Voluceau

Rocquencourt

$$
\text { B.P.105 }
$$

78153 Le Chesnay Cedex

$$
\text { France }
$$

Tél.:(1)39635511
Isaac COHEN

Nicholas AYACHE

Patrick SULGER 


\title{
Tracking Points on Deformable Objects
}

\section{Using Curvature Information}

\section{Suivi de Points sur des Objets Déformables en Utilisant la Courbure*}

\author{
Isaac COHEN! Nicholas AYACHE, Patrick SULGER \\ INRIA, Domaine de Voluceau, Rocquencourt, \\ B.P. 105, 78153 Le Chesnay CEDEX, France. \\ Email isaac@bora.inria.fr, na@bora.inria.fr \\ Phone: 1-39635366 - Fax: 1-396353 30 .
}

-This work was partially supported by Digital Equipment Corporation.

'Boursier MRT, Université Paris-IX Dauphine. 


\section{Abstract}

The objective of this paper is to present a significant improvement to the approach of Duncan et al. $[1,7]$ to analyze the deformations of curves in sequences of 2-D images. This approach is based on the paradigm that high curvature points usually possess an anatomical meaning, and are therefore good landmarks to guide the matching process, especially in the absence of a reliable physical or deformable geometric model of the observed structures.

As Duncan's team, we therefore propose a method based on the minimization of an energy which tends to preserve the matching of high curvature points, while ensuring a smooth field of displacement vectors everywhere.

The innovation of our work stems from the explicit description of the mapping between the curves to be matched, which ensures that the resulting displacement vectors actually map points belonging to the two curves, which was not the case in Duncan's approach. Moreover, the energy minimization is obtained through the mathematical framework of Finite Elements analysis, which provides a rigorous and efficient numerical solution. Moreover we show that the generalization of our approach can be easily generalized in 3-D to analyze the deformations of surfaces.

We have actually implemented the method in 2-D and we present the results of the tracking of a heart structure in a sequence of ultrasound images.

\section{Résumé}

L'objectif de cet article est de présenter une amélioration importante de la méthode présentée par Duncan et al. $[1,7]$ pour analyser la déformation de courbes dans une séquence d'images 2-D. Cette approche est basée sur le principe que les points de forte courbure ont une signification anatomique et par conséquent sont importants pour la mise en correspondance des points. De plus, cette approche n'est pas sous-jacente à un modèle physique ou géométrique des structures observées dans la séquence d'images.

Nous proposons, comme Duncan et al. [1,7], une méthode basée sur la minimisation d'une énergie qui préserve la mise en correspondance de points de forte courbure tout en assurant un champ de déplacement régulier.

L'innovation provient de la description explicite de l'application mettant en correspondance les courbes, ce qui garantit que le champ de déplacement met en correspondance des points appartenant aux deux courbes, contrairement à la méthode de Duncan. De plus, la minimisation de l'énergie est faite a l'aide d'une méthode variationnelle et d'éléments finis. La méthode présentée peut être également généralisée au suivi de déformations de surfaces dans des images 3-D.

Nous avons implémenté la méthode dans le cas de courbes 2-D, et nous présentons les résultats obtenus sur le suivi de la valve mitrale dans une séquence echocardiographique. 


\section{Introduction}

Non-rigid motion of deformable shapes is becoming an increasingly important topic in computer vision, especially for medical image analysis. Within this topic, we concentrate on the problem of tracking deformable objects through a time sequence of images.

The objective of our work is to improve the approach of Duncan et al. $[1,7]$ to analyze the deformations of curves in sequences of 2-D images. This approach is based on the paradigm that high curvature points usually possess an anatomical meaning, and are therefore good landmarks to guide the matching process. This is the case for instance when deforming patients skulls (see for instance $[6,8,16]$, or when matching patient faces taken at different ages, when matching multipatients faces, or when analyzing images of a beating heart. In these cases, many lines of extremal curvatures (or ridges) are stable features which can be reliably tracked between the images (on a face they will correspond to the nose, chin and eyebrows ridges for instance, on a skull to the orbital, sphenoid, falx, and temporal ridges, on a heart ventricle to the papillary muscle etc...).

As Duncan's team, we therefore propose a method based on the minimization of an energy which tends to preserve the matching of high curvature points, while ensuring a smooth field of displacement vectors everywhere.

The innovation of our work stems from the explicit description of the mapping between the curves to be matched, which ensures that the resulting displacement vectors actually map points belonging to the two curves, which was not the case in Duncan's approach. Moreover, the energy minimization is obtained through the mathematical framework of Finite Elements analysis, which provides a rigorous and efficient numerical solution. Moreover we show that the generalization of our approach can be easily generalized in 3-D to analyze the deformations of surfaces.

Our approach is particularly attractive in the absence of a reliable physical or deformable geometric model of the observed structures, which is often the case when studying medical images. When such a model is available, other approaches would involve a parametrization of the observed shapes [13], a modal analysis of the displacement field [11], or a parametrization of a subset of deformations $[2,14]$. In fact we believe that our approach can always be used when some sparse geometric features provide reliable landmarks, either as a preprocessing to provide an initial solution to the other approaches, or as a post-processing to provide a final smoothing which preserves the matching of reliable landmarks.

We have actually implemented the method in $2-D$ and we show how to generalize the approach 
in 3-D. We present the results of the tracking of a heart structure in a sequence of 2-D ultrasound images.

\section{Modelling the Problem}

Let $C_{P}$ and $C_{Q}$ be two boundaries of the image sequence, the contour $C_{Q}$ is obtained by a non rigid (or elastic) deformation of the contour $C_{P}$. The curves $C_{P}$ and $C_{Q}$ are parameterized by $P(s)$ and $Q\left(s^{\prime}\right)$ respectively.

The problem is to determine for each point $P$ on $C_{P}$ a corresponding point $Q$ on $C_{Q}$. For doing this, we must define a similarity measure which will compare locally the neighborhoods of $P$ and $Q$.

As explained in the introduction, we assume that points of high curvature correspond to stable salient regions, and are therefore good landmarks to guide the matching of the curves. Moreover, we can assume as a first order approximation, that the curvature itself remains invariant in these regions. Therefore, we can introduce an energy measure in these regions of the form:

$$
E_{\text {curve }}=\frac{1}{2} \int_{\delta S}\left(K_{Q}\left(s^{\prime}\right)-K_{P}(s)\right)^{2} d s
$$

where $K_{P}$ and $K_{Q}$ denote the curvatures and $s, s^{\prime}$ parameterize the curves $C_{P}$ and $C_{Q}$ respectively. In fact, as shown by $[7,12]$, this is proportional to the energy of deformation of an isotropic elastic planar curve.

We also wish the displacement field to vary smoothly around the curve, in particular to insure a correspondence for points lying between two salient regions. Consequently we consider the following functional (similar to the one used by Hildreth to smooth a vector flow field along a contour [10]) :

$$
E=\int_{C_{P}}\left(K_{Q}\left(s^{\prime}\right)-K_{P}(s)\right)^{2} d s+R \int_{C_{P}}\left\|\frac{\partial\left(Q\left(s^{\prime}\right)-P(s)\right)}{\partial s}\right\|^{2} d s
$$

where

$$
E_{\text {regular }}=\int_{C_{P}}\left\|\frac{\partial\left(Q\left(s^{\prime}\right)-P(s)\right)}{\partial s}\right\|^{2} d s
$$

measures the variation of the displacement vector $\overrightarrow{P Q}$ along the curve $C_{P}$, and the $\|$.$\| denotes the$ norm associated to the euclidean scalar product $\langle. .$.$\rangle in the space R^{2}$.

The regularization parameter $R(s)$ depends on the shape of the curve $C_{P}$. Typically, $R$ is inversely proportional to the curvature at $P$, to give a larger weight to $E_{\text {curve }}$ in salient regions and conversely to $E_{\text {regular }}$ to points inbetween. This is done continuously without annihiling totally the weight of any of these two energies (see section 5.2). 


\section{Mathematical Formulation of the Problem}

Given two curves $C_{P}$ and $C_{Q}$ parameterized by $s \in[0,1]$ and $s^{\prime} \in[0, \alpha]$ (where $\alpha$ is the length of the curve $C_{Q}$ ), we have to determine a function $f$ such that:

$$
\begin{gathered}
f:[0,1] \rightarrow[0, \alpha] \\
s \rightarrow s^{\prime}
\end{gathered}
$$

satisfying

$$
f(0)=0 \text { and } f(1)=\alpha
$$

and

$$
f=\operatorname{Arg} M i n(E(f))
$$

where

$$
E(f)=\int_{C_{P}}\left(K_{Q}(f(s))-K_{P}(s)\right)^{2} d s+R \int_{C_{P}}\left\|\frac{\partial(Q(f(s))-P(s))}{\partial s}\right\|^{2} d s
$$

The condition (3) means that the displacement vector is known for one point of the curve. In the trivial case, where the curves $C_{P}$ and $C_{Q}$ are identical, solving (4) leads to the identity function.

In the model defined above we assumed that:

- the boundaries have already been extracted,

- the curvatures $K$ are known on the pair of contours.

These necessary data are obtained by preprocessing the image sequence.

\subsection{Boundaries Extraction}

The boundaries of the object are obtained with the deformable model [5]. This produces a good boundary extraction, the detected edges are connected and have an accurate localization. The deformable model allows also to do a global boundary tracking. This last feature is important in processing an image sequence, since in this case the deformable model has to be initialized only once on the first image of the temporal sequence. 


\subsection{The Computation of the Curvature}

The computation of the curvatures of the two curves $C_{P}$ and $C_{Q}$ is done by fitting a fourth order $\mathrm{B}$-spline (insuring a $\mathcal{C}^{3}$ continuity) to the extracted boundaries. The control points of the B-spline are distributed in a non uniform manner, insuring a good localization of the control points near the points of interest (typically high curvature points). The details of this method are given in [8].

\section{Numerical Solution}

The characterization of a function $f$ satisfying $f=\operatorname{Arg} M i n(E(f))$ and the condition (3) is performed by a variational method. This method characterizes a local minimum $f$ of the functional $E(f)$ as the solution of the Euler-Lagrange equation $\nabla E(f)=0$.

Then solving the Euler-Lagrange equation $\nabla E(f)=0$ leads to the solution of the partial differential equation:

$$
\left\{\begin{array}{l}
f^{\prime \prime}\left\|Q^{\prime}(f)\right\|^{2}+K_{P}\left\langle N_{P}, Q^{\prime}(f)\right\rangle+\frac{1}{R}\left[K_{P}-K_{Q}(f)\right] K_{Q}^{\prime}(f)=0 \\
+ \text { Boundary conditions }
\end{array}\right.
$$

where $Q$ is a parametrization of the curve $C_{Q}, Q^{\prime}(f)$ the tangent vector of $C_{Q}, K_{Q}^{\prime}$ the derivative of the curvature of the curve $C_{Q}$ and $N_{P}$ is the normal vector to the curve $C_{P}$. The boundary conditions of equation (6) are $f(0)=0$ and $f(1)=\alpha$ (condition (3)) that any solution must satisfy. In the following we consider null boundary conditions (this is done by a simple change of variables).

The equation (6) is solved by a finite element method. Details on the associated variational problem and the construction of the approximation space can be found in the appendix.

The term $\int_{C_{P}}\left(K_{Q}(f(s))-K_{P}(s)\right)^{2} d s$ measures the difference between the curvature of the two curves. This induces a non convexity of the functional $E$. Consequently, solving the partial differential equation (6) will give us a local minimum of $E$. To overcome this problem we will assume that we have an initial estimation $f_{0}$ which is a good approximation of the real solution (the definition of the initial estimation $f_{0}$ will be explained later). This initial estimation defines a starting point for the search of a local minimum of the functional $E$. To take into account this initial estimation we consider the associated evolution equation:

$$
\left\{\begin{array}{l}
\frac{\partial f(s)}{\partial t}+f^{\prime \prime}(s)\left\|Q^{\prime}(f(s))\right\|^{2}+K_{P}(s)\left\langle N_{P}(s), Q^{\prime}(f(s))\right\rangle+\frac{1}{R}\left[K_{P}(s)-K_{Q}(f(s))\right] K_{Q}^{\prime}(f(s))=0 \\
f(0, s)=f_{0}(s) \text { initial estimation. }
\end{array}\right.
$$


A stationary solution of the equation (7) is a solution of (6), since, at a stationary state, the term $\frac{\partial f}{\partial t}$ vanishes and the obtained solution satisfies the equation (6).

This evolution equation can also be seen as a gradient descent algorithm toward a minimum of the energy $E$.

\subsection{Discrete Problem}

The discretization of the equation (6) by a finite element method leads to a solution of a linear system $A f=L$, where the matrix $A$ is symmetric, positive definite and tridiagonal. Thus the evolution problem (7) can be rewritten as:

$$
\frac{\partial f}{\partial t}+A f=L_{f}
$$

where the vector $L_{f}$ depend on the current solution $f$. This equation is discretized by a finite difference method and leads to the solution of the linear system:

$$
\left\{\begin{array}{l}
\left(I_{d}+\tau A\right) f_{t}=f_{t-1}+\tau L_{f_{t-1}} \\
f_{t=0}=f_{0}
\end{array}\right.
$$

where $\tau$ is the time step.

Thus at each time step we solve a symmetric, positive definite, tridiagonal linear system by a LU decomposition.

The scheme (9) is iterated as long as $\left\|f_{t}-f_{t-1}\right\|$ is greater than a given value $\epsilon>0$.

\subsection{Determining the Initial Estimation $f_{0}$}

The definition of the initial estimation $f_{0}$ has an effect upon the convergence of the algorithm. Consequently a good estimation of the solution $f$ will lead to a fast convergence. The definition of $f_{0}$ is based on the work of Duncan et al [7]. The method is as follows:

Let $s_{i} \in[0,1], i=1 \ldots n$ be a subdivision of the interval $[0,1]$.

For every point $P_{i}=\left(X\left(s_{i}\right), Y\left(s_{i}\right)\right)$ of the curve $C_{P}$ we search for a point $Q_{i}=\left(X\left(s_{i}^{\prime}\right), Y\left(s_{i}^{\prime}\right)\right)$ on the curve $C_{Q}$, and the function $f_{0}$ is then defined by $f_{0}\left(s_{i}\right)=s_{i}^{\prime}$.

For doing so we have to define a pair of points $P_{0}, Q_{0}$ which correspond to each other. But, first of all, let us describe the search method. In the following, we identify a point and its curvilinear abscissa (i.e. the point $s_{i}$ denotes the point $P_{i}$ of the curve $C_{P}$ such that $P\left(s_{i}\right)=P_{i}$, where $P$ is the parametrization of the curve $C_{P}$ ). 
For each point $s_{i}$ of $C_{P}$ we associate a set of candidates $S_{i}$ on the curve $C_{Q}$. The set $S_{i}$ defines the search area. This set is defined by the point $s^{\prime}$ which is the nearest distance point to $s_{i}$ belonging to the curve $C_{Q}$, along with $\frac{N_{\text {aearch }}-1}{2}$ points of the curve $C_{Q}$ on each side of $s^{\prime}$ (where $N_{\text {search }}$ is a given integer defining the length of the search area).

The length of the search area plays an important role in the determination of $f_{0}$. The parameter $N_{\text {search }}$ depends on the shape of the curves $C_{P}$ and $C_{Q}$. If the curve $C_{Q}$ is similar to $C_{P}$ (i.e. if $C_{P}$ undergoes small deformations) then $N_{\text {search }}$ can be small. But in the opposite case $N_{\text {search }}$ must be large enough to include the corresponding points of $C_{P}$ and $C_{Q}$. In the presented experimental results we have set $N_{\text {search }}=10$.

Among these candidates, we choose the point which minimizes the deformation energy:

$$
E=\frac{1}{2} \int_{\delta_{s_{i}}}\left(K_{Q}(f(s))-K_{P}(s)\right)^{2} d s
$$

where $\delta_{S_{i}}=\left[s_{i}-\frac{\delta}{2}, s_{i}+\frac{\delta}{2}\right]$ and $\delta$ denotes the length of a characteristic structure.

The length of the characteristic structure depends on the shape of the curve $C_{P}$. If the curve $C_{P}$ has small structures (such that corners) the parameter $\delta$ must be small, not to smooth these structures by computing $E(10)$ over a large set $\delta S_{i}$. This parameter allows to do a local matching process between the curves $C_{P}$ and $C_{Q}$.

In some situations this method fails, and the obtained estimation $f_{0}$ is meaningless, leading to a bad solution. Figure 9 shows an example where the method described in [7] fails. This is due to the bad computation of the search area $S_{i}$. To compute more accurately this set, we have added a criterion based on the curvilinear abscissa. Consequently, the set defining the search area $S_{i}$ is defined by the point $s^{\prime}$ which is the nearest distance point to $s_{i}$ belonging to the curve $C_{Q}$ such that $s_{i}^{\prime} \approx \frac{s_{i}}{\alpha}$, along with $\frac{N_{\text {eearch }}-1}{2}$ points of the curve $C_{Q}$ on each side of $s_{i}^{\prime}$.

Figure 10 illustrates the use of this new definition of the set $S_{i}$ for the same curves given in figure 9. This example shows the ability to handle more general situations with this new definition of the search area $S_{i}$.

As noted above, the search area $S_{i}$ can be defined only if we have already chosen a point $P_{0}$ and its corresponding point $Q_{0}$. The most salient features in a temporal sequence undergo small deformations at each time step, thus a good method for choosing the point $P_{0}$ is to take the most distinctive point so that the search for the corresponding point becomes a trivial task. Consequently the point $P_{0}$ is chosen among the points of $C_{P}$ with maximal curvature. In many cases this method provides a unique point $P_{0}$. Once we have chosen the point $P_{0}$, the point $Q_{0}$ is found by the local 
search described above.

\section{Defining the Parameters}

In this section we discuss the choice of the parameters used in our model. The parameters are: the space discretization step $h$, the time step $\tau$ and the regularization parameter $R=R_{C_{P}}(s)$.

\subsection{The Space and Time Steps $h$ and $\tau$}

We have shown that solving equation (7) leads to a solution of a linear system (9). This system is solved at each time step $\tau$ and the accuracy of the solution depends on the value of $\operatorname{Cond}\left(I_{d}+\tau A\right)=$ $\left\|I_{d}+\tau A\right\|\left\|\left(I_{d}+\tau A\right)^{-1}\right\|$ which is the condition number of the matrix $\left(I_{d}+\tau A\right)$. In the optimal case $\operatorname{Cond}\left(I_{d}+\tau A\right)=1$. In practice we will choose the parameters $h$ and $\tau$ such that $C o n d\left(I_{d}+\tau A\right)$ is closest to one. An easy way to do so is to search for an upper bound of $\operatorname{Cond}\left(I_{d}+\tau A\right)$ and then choose the space and step discretization steps such that the upper bound is closest to one.

Let us consider the matrix norm:

$$
\left\|\left(a_{i j}\right)_{i, j=1 \ldots n} \mid\right\|=\frac{1}{\sqrt{n}}\left(\sum_{i} S u p_{j}\left|a_{i j}\right|^{2}\right)^{\frac{2}{2}}
$$

thus,

$$
\operatorname{Cond}\left(I_{d}+\tau A\right) \leq 1+\frac{n}{n+2}\left(\frac{\beta}{1+\beta}\right)^{2}
$$

where $n$ is the dimension of the matrix $A$ and $\beta=\frac{6 \tau}{5 h}$ is the diagonal element of the matrix $A$ ( $\beta$ is obtained by the finite element method).

Consequently, choosing $\beta \simeq 1$ which means that $h$ and $\tau$ have similar values, leads to:

$$
\operatorname{Cond}\left(I_{d}+\tau A\right)<1.12
$$

insuring that the matrix $I_{d}+\tau A$ is well-conditioned.

\subsection{The Regularization Parameter $R=R_{C_{P}}(s)$}

The energy $E(5)$ is defined by an energy of deformation $E_{\text {curve }}$ which measures the difference between the curvatures of $C_{P}$ and $C_{Q}$ and a regularization energy $E_{\text {regular }}$ which constrains the solution $f$ to be smooth. The balance between these two energies is defined by the regularization parameter $R_{C_{P}}(s)$. 
The parameter $R\left(C_{P}\right)$ allows the algorithm to deal with curves which have locally a constant curvature. In this case the energy $E_{\text {curve }}$ is locally constant and the regularization parameter $R\left(C_{P}\right)$ constrains the solution to be smooth. In this case setting $R\left(C_{P}\right)=0$ the energy $E$ is locally constant and consequently any function $f \in H^{1}(0,1)$ satisfies $\nabla E(f)=0$, leading to a degenerate case.

Experimenting the algorithm with different types of curves gave us an empirical method for choosing the regularization parameter $R\left(C_{P}\right)$. If the curves $C_{P}$ and $C_{Q}$ have some characteristic points (typically points of high curvature), one then choose $R\left(C_{P}\right)$ such that the energy $E_{\text {curve }}$ is preponderant. In the opposite case, i.e if the curvatures $K_{P}$ and $K_{Q}$ are nearly constant (in this case we have the situation described above), one must choose the parameter $R\left(C_{P}\right)$ such that the energy $E_{\text {regular }}$ is preponderant.

In both cases the parameter $R\left(C_{P}\right)$ should not have the extremal values $R\left(C_{P}\right)=0$ or $R\left(C_{P}\right) \gg 1$. If $R\left(C_{P}\right)=0$ then each point matches the point of closest curvature, leading to function $f$ very rough. Setting $R\left(C_{P}\right) \gg 1$ leads to an excessive regularization and the obtained solution will be the identity function.

In the presented experimental results we set $R\left(C_{P}\right)=\frac{1}{1+K_{P}(s)^{2}}$.

\section{Experimental Results}

The method was tested on a set of synthetic and real image sequences. The results are given by specifying at each discretization point $P_{i} i=1 \ldots N$ of the curve $C_{P}$ the displacement vector $\overrightarrow{u_{i}}=P_{i} \vec{Q}_{i}$. At each point $P_{i}$ the arrow represents the displacement vector $\overrightarrow{u_{i}}$.

The first experiments were made on synthetic data. In figure 3 , the curve $C_{Q}$ (a square) is obtained by a similarity transformation (translation, rotation and scaling) of the curve $C_{P}$ (a rectangle). The initial estimation $f_{0}$ is shown in figure 3 , and the solution after a few iterations of the scheme (9) is given in figure 4. We can note that the algorithm computes accurately the displacements of the four corners. This result was expected since the curves $C_{P}$ and $C_{Q}$ have salient features which help the algorithm to compute accurately the displacement vector $\overrightarrow{u_{i}}$.

Figure 5 and 6 give an example of the tracking of each point on an ellipse deformed by a similarity. This figure illustrates the robustness of the algorithm, since in this example, locally, the curvature is modified by the similarity transformation.

These two examples characterize the algorithm: the displacement vectors are computed accu- 
rately when, locally, the curvature of the curve $C_{P}$ varies significantly, otherwise the regularization energy prevails over the deformation energy and smoothes the solution.

As described in section (4.2) the computation of the initial estimation is crucial. In the following experimentation we have tried to define the maximal error that can be done on the estimation of $f_{0}$ without disturbing the final result. In figure 7 we have added a gaussian noise $(\sigma=0.05)$ to a solution $f$ obtained by the scheme (9). This noisy function was taken as an initial estimation for the iterative scheme (9). After a few iterations the solution $f$ is recovered (8). It appears that if $\left|f-f_{0}\right| \leq 4 h$ (where $h$ is the space discretization step), starting with $f_{0}$ the iterative scheme (9) will converge toward the solution $f$. The inequality $\left|f-f_{0}\right| \leq 4 h$ means that for each point $P$ on the curve $C_{P}$ the corresponding point $Q$ can be determined with an error of 4 points over the grid of the curve $Q$.

The tracking of the moving boundaries of the valve of the left ventricle on an ultrasound image helps to diagnose some heart diseases. The figures $12,13,14$ and 15 show a temporal tracking of each point of the valve during a cardiac cycle. The segmentation of the moving boundaries over the whole sequence was done by the snake model [5]. Figure (2,left) shows the initial estimation of the boundary. This estimation is given by the user once on the first image of the temporal sequence, the rest of the sequence is processed almost automatically [9]. The user gave 4 initial estimations for the whole temporal sequence ( 30 images) due to the movements of the valve which undergoes big deformations at some characteristics periods of the cardiac cycle. Figure (2,right) shows the obtained segmentation of the boundary of the first image. In figure 11 a global tracking of a part of the image sequence is showed ${ }^{1}$. This set of curves are processed as described in section (3.2) to obtain the curvatures and the normal vector of the curves. The figures $12,13,14$ and 15 show the tracking of some points of the valve during the whole temporal sequence. The results are presented by pairs of successive contours. One can visualize that the results meet perfectly the objectives of preserving the matching of high curvature points while insuring a smooth displacement field.

\section{3-D Generalization}

In this section we give a 3-D generalization of the algorithm described in the previous sections. In 3-D imaging we must track points on located surfaces, since the objects boundaries are surfaces (as in [1]).

\footnotetext{
${ }^{2}$ Courtesy of I. Herlin [9]
} 
In [15] the authors have shown on a set of experimental data, that the extrema of the larger principal curvature often correspond to significant intrinsic (i.e. invariant by the group of rigid transformation) features which might characterize the surface structure, even in the presence of small anatomic deformations.

Let $S_{P}$ and $S_{Q}$ be two surfaces parameterized by $P(s, r)$ and $Q\left(s^{\prime}, r^{\prime}\right)$, and let $\kappa_{P}$ denote the larger value of the principal curvature of the surface $S_{P}$ at point $P$.

Thus the matching of two surfaces, leads to the following problem:

find a function

$$
\begin{gathered}
f: R^{2} \rightarrow R^{2} \\
(s, r) \mapsto\left(s^{\prime}, r^{\prime}\right) .
\end{gathered}
$$

which minimizes the functional:

$$
\begin{aligned}
E(f)= & \int_{S_{P}}\left(\kappa_{Q}(f(s, r))-\kappa_{P}(s, r)\right)^{2} d s d r \\
& +R, \int_{S_{P}}\left\|\frac{\partial(Q(f(s, r))-P(s, r))}{\partial s}\right\|^{2} d s d r+R_{r} \int_{S_{P}}\left\|\frac{\partial(Q(f(s, r))-P(s, r))}{\partial r}\right\|^{2} d s d r
\end{aligned}
$$

where $\|$.$\| denotes the euclidean norm in R^{3}$. Its resolution by a finite element technique can be done as in [4], and the results should be compared to those obtained by [1]. We are currently working on the implementation of this 3-D method.

\section{Conclusion}

We presented a significant improvement to Duncan's team approach to track the motion of deformable 2-D shapes, based on the tracking of high curvature points while preserving the smoothness of the displacement field. This approach is an alternative to the other approaches of the literature, when no physical or geometric model is available, and can also be used as a complementary approach otherwise.

The results on a real sequence of time varying anatomical structure of the beating heart perfectly meet the defined objectives.

Our future work will concentrate on the 3-D generalization of this approach. 


\section{Appendices}

In this section we give the details about the minimization of the energy (5). This minimization is done in three stages. First, establish the partial differential equation from the Euler-Lagrange equation. Second, construct the associated variational problem and then solve this problem with a finite element method [3].

\subsection{Euler-Lagrange Equation}

A local minimum of $E(5)$ satisfies the Euler-Lagrange equation: $\nabla E=\nabla E_{\text {curve }}+R \nabla E_{\text {regular }}=0$ For any function $u$, we have:

$$
\nabla E_{\text {curve }}(f) \cdot u=-2 \int_{C_{P}}\left[K_{P}(s)-K_{Q}(f(s))\right] K_{Q}^{\prime}(f(s)) u d s
$$

and

$$
\begin{aligned}
\nabla E_{\text {regular }}(f) \cdot u & =-2 \int_{C_{P}}\left\langle\frac{\partial(P(s)-Q(f(s)))}{\partial s}, \frac{\partial}{\partial s}\left(Q^{\prime}(f(s)) u(s)\right)\right\rangle d s \\
& =-2 \int_{C_{P}}\left\langle\frac{\partial^{2}(Q(f(s))-P(s))}{\partial s^{2}}, Q^{\prime}(f(s)) u(s)\right\rangle d s \\
& =-2 \int_{C_{P}}\left\langle f^{\prime \prime}(s) Q^{\prime}(f(s))+f^{\prime}(s) Q^{\prime \prime}(f(s))-P^{\prime \prime}(s), Q^{\prime}(f(s))\right\rangle u(s) d s
\end{aligned}
$$

$Q$ is a parametrization of the curve $C_{Q}$ thus $\left\langle Q^{\prime}, Q^{\prime \prime}\right\rangle=0$ (where the $\langle.,$.$) denotes the euclidean$ scalar product in $R^{2}$ and $\|\cdot\|$ the associated norm). We have also the relation

$$
P^{\prime \prime}(s)=-K_{P}(s) N_{P}(s)
$$

where $P^{\prime \prime}$ denotes the second derivatives of $P, K_{P}$ the curvature and $N_{P}$ the normal vector to the curve $C_{P}$. Hence,

$$
\nabla E_{\text {regular }}(f) \cdot u=-2 \int_{C_{P}}\left(f^{\prime \prime}(s)\left\|Q^{\prime}(f(s))\right\|^{2}+K_{P}(s)\left\langle N_{P}(s), Q^{\prime}(f(s))\right\rangle\right) u(s) d s
$$

Since the equations $(11,12)$ are satisfied, independently of the function $u$, we have the partial differential equation:

$$
\left\{\begin{array}{l}
f^{\prime \prime}\left\|Q^{\prime}(f)\right\|^{2}+K_{P}\left\langle N_{P}, Q^{\prime}(f)\right\rangle+\frac{1}{R}\left[K_{P}-K_{Q}(f)\right] K_{Q}^{\prime}(f)=0 \\
+ \text { Boundary conditions }
\end{array}\right.
$$

given in section 4 . 


\subsection{The Variational Problem}

A method for solving the equation (13) is to consider the associate variational problem. This consists in defining a bilinear form $a(f, g)$ and a linear form $L$ such that solving the equation (13) is equivalent to solve:

find a function $f$ such that:

$$
a(f, g)=L(g) \quad \forall g \in H^{1}(0,1)
$$

$\left(H^{1}(0,1)\right.$ is the space of functions $v \in L^{2}(0,1)$ such that $\left.\int_{0}^{1}\left(\frac{d v}{d s}\right)^{2} d s<+\infty\right)$.

Let $g \in H^{1}(0,1)$, multiplying equation (13) by $g$ and integrating it, yields:

$$
\int_{0}^{1}\left\|Q^{\prime}(f)\right\|^{2} f^{\prime} g^{\prime} d s=\int_{0}^{1}\left(K_{P}\left\langle N_{P}, Q^{\prime}(f)\right\rangle+\frac{1}{R}\left[K_{P}-K_{Q}(f)\right] K_{Q}^{\prime}(f)\right) g d s
$$

The bilinear form is then defined by:

$$
a(f, g)=\int_{0}^{1}\left\|Q^{\prime}(f)\right\|^{2} f^{\prime} g^{\prime} d s
$$

and the linear form $L$ is given by:

$$
L(g)=\int_{0}^{1}\left(K_{P}\left\langle N_{P}, Q^{\prime}(f)\right\rangle+\frac{1}{R}\left[K_{P}-K_{Q}(f)\right] K_{Q}^{\prime}(f)\right) g d s
$$

One can easily check that $f$ is a solution of equation (13) if and only if $f$ is a solution of the variational problem (14).

The variational problem (14) has a unique solution since the bilinear form $a(f, g)$ is continuous, $H^{1}$-elliptic and $L$ is continuous.

\subsection{Obtaining the Discrete Problem: The Finite Element Method}

So far, we have been dealing with the continuous form of the equation (13) to show the existence and the uniqueness of the solution. Here, we will use the Galerkin's method to approximate the variational problem (14) by a discrete problem defined over a finite dimensional subspace $V_{h} \subset H^{1}(0,1)$. The associated discrete problem is:

Find $f_{h} \in V_{h}$ such that $a\left(f_{h}, g_{h}\right)=L\left(g_{h}\right) \quad \forall g_{h} \in V_{h}$.

To insure the convergence of $f_{h}$ toward $f$ (solution of 14), we construct the space $V_{h}$ with the finite element method. 
Let consider a subdivision $\bigcup_{i=1}^{N}[i h,(i+1) h]$ of the set $[0,1]$ and a basis function $\left\{\phi_{i}\right\}_{i=1} \ldots N$ of the subspace $V_{h}$ (see figure 1). The space $V_{h}$, generated by these basis functions, fulfil the necessary conditions of the finite element method.

Thus $\forall f_{h} \in V_{h}$ we have $f_{h}=\sum_{i=1}^{N} f_{h}(i h) \phi_{i}$.

Using this relation the discrete problem (15) can be reformulated as:

$$
\text { Find } f_{h}(i h), i=1 \ldots N \text { such that } \sum_{i=1}^{N} f_{h}(i h) a\left(\phi_{i}, g_{h}\right)=L\left(g_{h}\right) \quad \forall g_{h} \in V_{h}
$$

This equation (16) holds also for the basis functions $\left\{\phi_{i}\right\}_{i=1 \ldots N}$, hence the discrete variational problem can be rewritten as

$$
\text { Find } f_{h}(i h), i=1 \ldots N \text { such that } \sum_{i=1}^{N} f_{h}(i h) a\left(\phi_{i}, \phi_{j}\right)=L\left(\phi_{j}\right) \quad \forall j=1 \ldots N .
$$

This gives a linear system $A \cdot F=L$ to solve, where $A=\left(a\left(\phi_{i}, \phi_{j}\right)\right)_{i j=1 \ldots N}, F=\left(f_{h}(i h)\right)_{i=1 \ldots N}$ and $L=\left(L\left(\phi_{i}\right)\right)_{i=1 \ldots N}$. The solution of the linear system $A \cdot F=L$ is done by a $L U$ decomposition since the matrix $A$ is positive definite, symmetric and tridiagonal.

\section{References}

[1] A. Amini, R. Owen, L. Staib, P. Anandan, and J. Duncan. non-rigid motion models for tracking the left ventricular wall. Lecture notes in computer science: Information processing in medical images. 1991. Springer-Verlag.

[2] Fred L. Bookstein. Principal warps: Thin-plate splines and the decomposition of deformations. IEEE Transactions on Pattern Analysis and Machine Intelligence, PAMI-11(6):567-585, June 1989.

[3] P. G. Ciarlet. The finite element methods for elliptic problems. NORTH-HOLLAND, 1987.

[4] Isaac Cohen, Laurent D. Cohen, and Nicholas Ayache. Using deformable surfaces to segment 3D images and infer differential structures. Computer Vision, Graphics, and Image Processing: Image Understanding, 1992. In press.

[5] Laurent D. Cohen and Isaac Cohen. A finite element method applied to new active contour models and 3-D reconstruction from cross sections. In Proc. Third International Conference on Computer Vision, pages 587-591. IEEE Computer Society Conference, December 1990. Osaka, Japan. 
[6] Court B. Cutting. Applications of computer graphics to the evaluation and treatment of major craniofacial malformation. In Jayaram K.Udupa and Gabor T. Herman, editors, 3-D Imaging in Medicine. CRC Press, 1989.

[7] J.S. Duncan, R.L. Owen, L.H. Staib, and P. Anandan. Measurement of non-rigid motion using contour shape descriptors. In Proc. Computer Vision and Pattern Recognition, pages 318-324. IEEE Computer Society Conference, June 1991. Lahaina, Maui, Hawaii.

[8] A. Guéziec and N. Ayache. Smoothing and matching of 3D-space curves. In Proceedings of the Second European Conference on Computer Vision (ECCV) 1992, Santa Margherita Ligure, Italy, May 1992.

[9] I.L. Herlin and N. Ayache. Features extraction and analysis methods for sequences of ultrasound images. In Proceedings of the Second European Conference on Computer Vision (ECCV) 1992, Santa Margherita Ligure, Italy, May 1992.

[10] Ellen Catherine Hildreth. The Measurement of Visual Motion. The MIT Press, Cambridge, Massachusetts, 1984.

[11] Bradley Horowitz and Alex Pentland. Recovery of non-rigid motion and structures. In Proc. Computer Vision and Pattern Recognition, pages 325-330. IEEE Computer Society Conference, June 1991. Lahaina, Maui, Hawaii.

[12] L.D. Landau and E.M. Lifshitz. Theory of elasticity. Pergamon Press, Oxford, 1986.

[13] Dimitri Metaxas and Demetri Terzopoulos. Constrained deformable superquadrics and nonrigid motion tracking. In Proc. Computer Vision and Pattern Recognition, pages 337-343. IEEE Computer Society Conference, June 1991. Lahaina, Maui, Hawaii.

[14] Sanjoy K. Mishra, Dmitry B. Goldgof, and Thomas S. Huang. Motion analysis and epicardial deformation estimation from angiography data. In Proc. Computer Vision and Pattern Recognition, pages 331-336. IEEE Computer Society Conference, June 1991. Lahaina, Maui, Hawaii.

[15] O. Monga, N. Ayache, and P. Sander. From voxel to curvature. In Proc. Computer Vision and Pattern Recognition, pages 644-649. IEEE Computer Society Conference, June 1991. Lahaina, Maui, Hawaii. 
[16] Olivier Monga, Serge Benayoun, and Olivier D. Faugeras. Using third order derivatives to extract ridge lines in 3-D images. In submitted to IEEE Conference on Vision and Pattern Recognition, Urbana Champaign, June 1992.

\section{Figures}

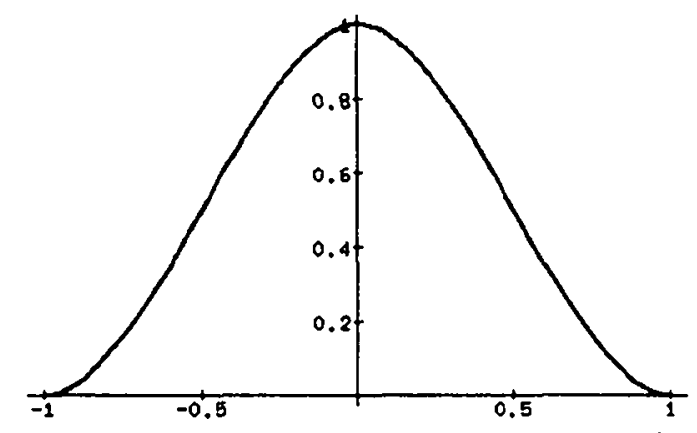

Figure 1: The finite element basis function used to discretize the continuous variational problem.
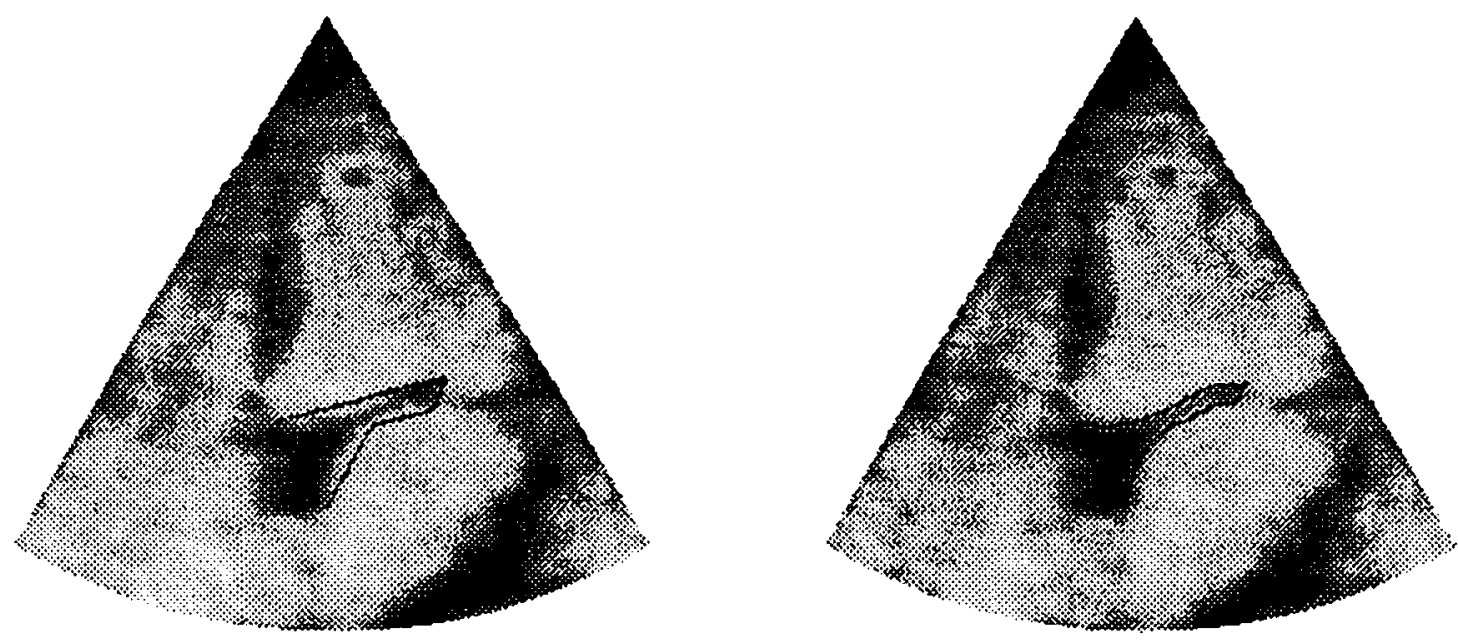

Figure 2: A example of processing the image sequence by a snake model to extract the boundaries of the moving object. Left: the initial estimation given by the user. Right: the boundary of the valve once the snake model have converged. The initial estimation is given only 4 times for a temporal sequence containing 30 images. 

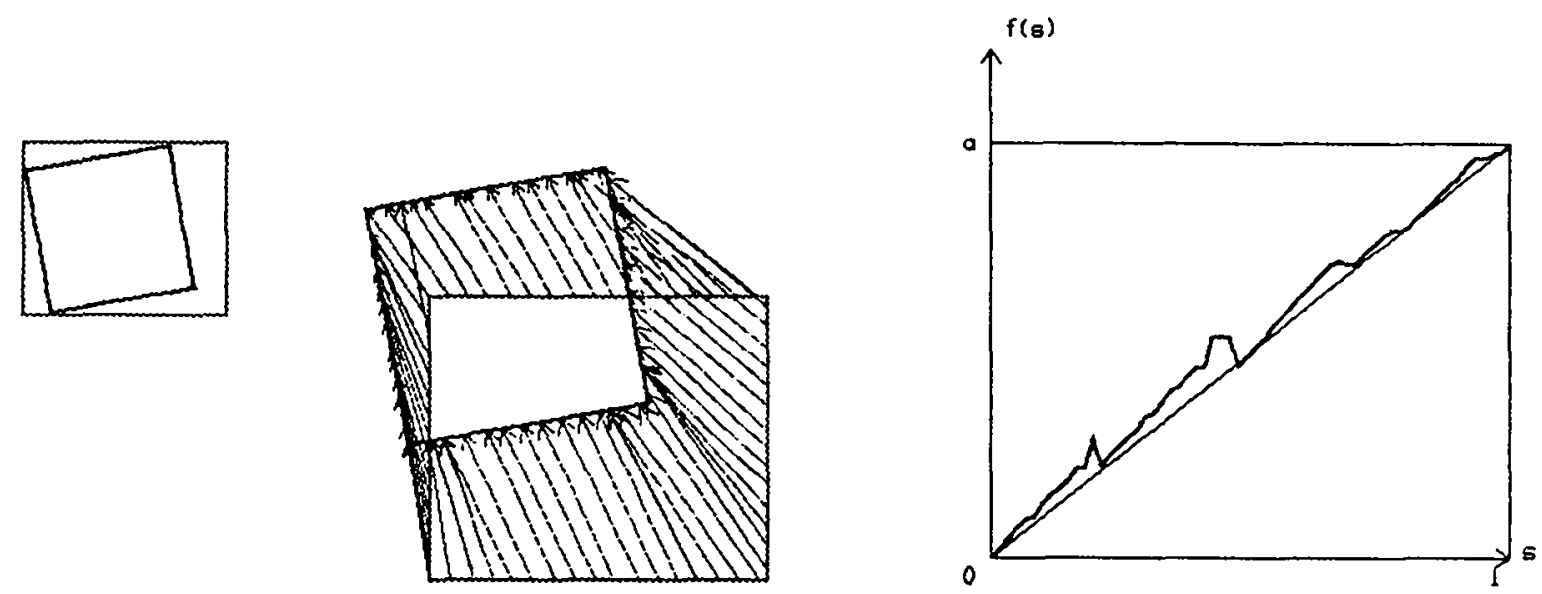

Figure 3: The rectangle (in grey) is deformed by a similarity (translation, rotation and scaling) to obtain the black square. In this figure we represent the initial estimation of the displacement vector of the curves (left) and the plotting of the function $f_{0}$ (right).
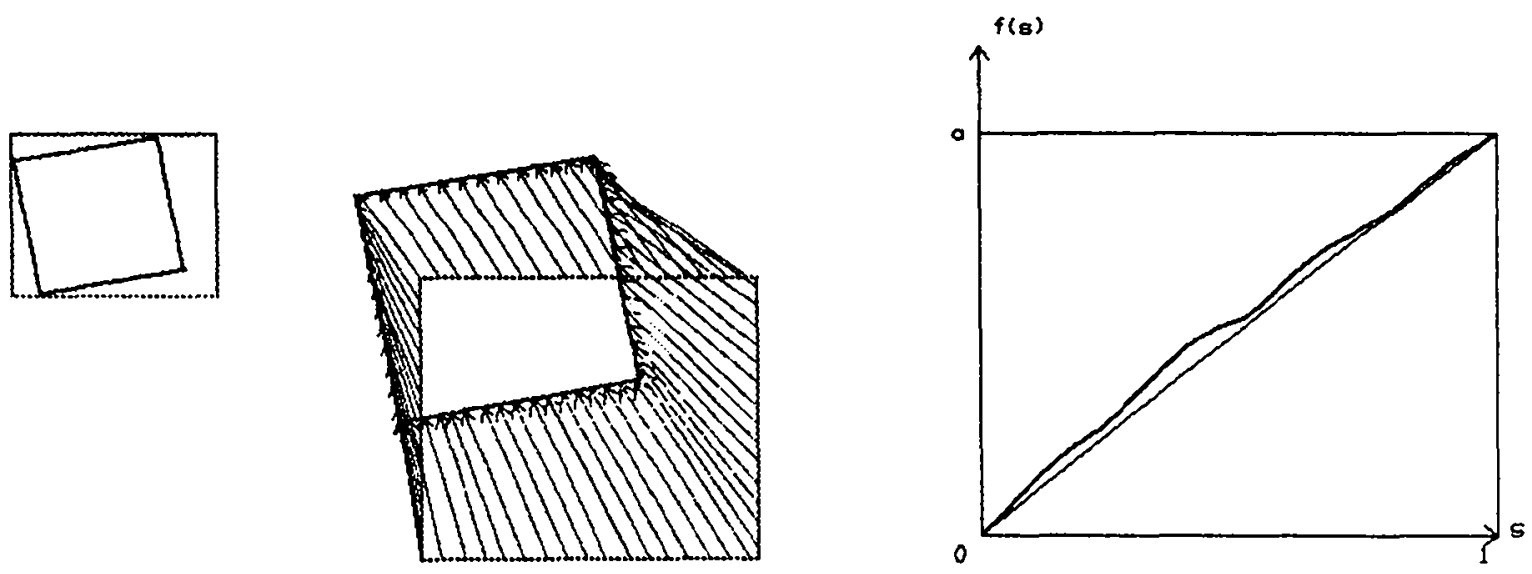

Figure 4: A representation (displacement field and the plotting) of the solution obtained by the algorithm. We can note that the displacement of the four corners is computed accurately. 

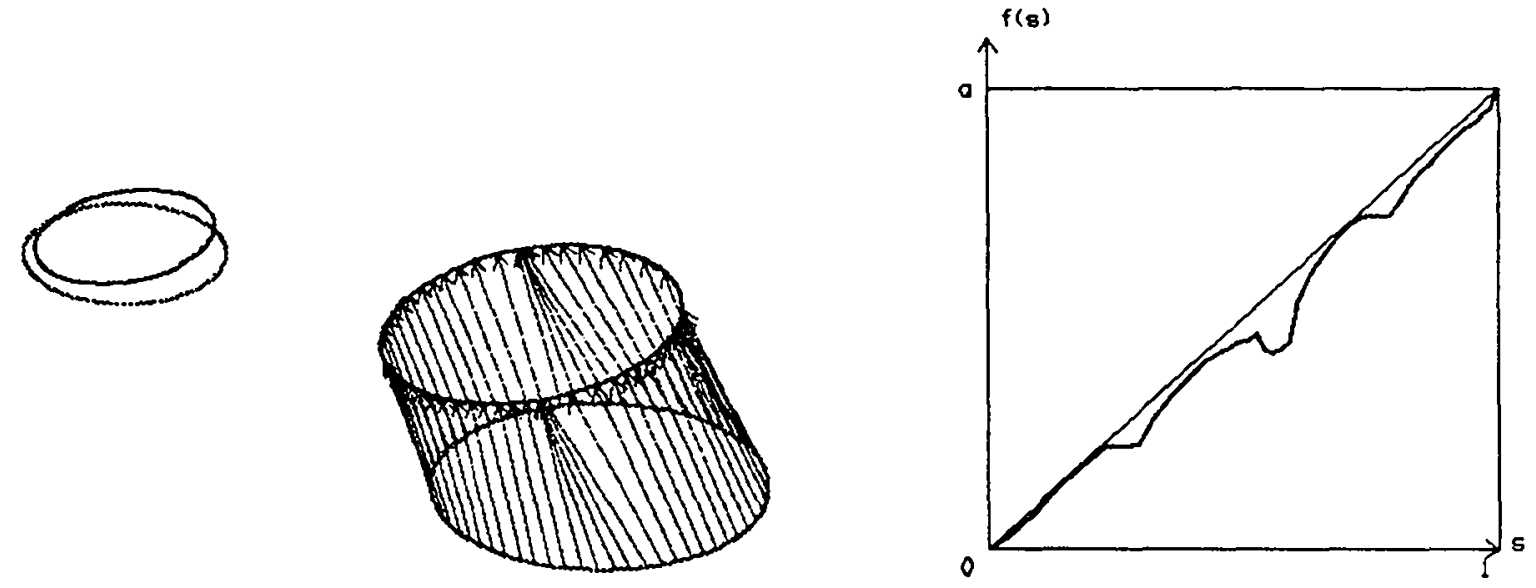

Figure 5: Another synthetic example, in this case the curvature along the curves $C_{P}$ and $C_{Q}$ varies smoothly. This often produces as a consequence in the computation of $f_{0}$ that several points of the curve $C_{P}$ (in grey) match the same point of $C_{Q}$ (in black).
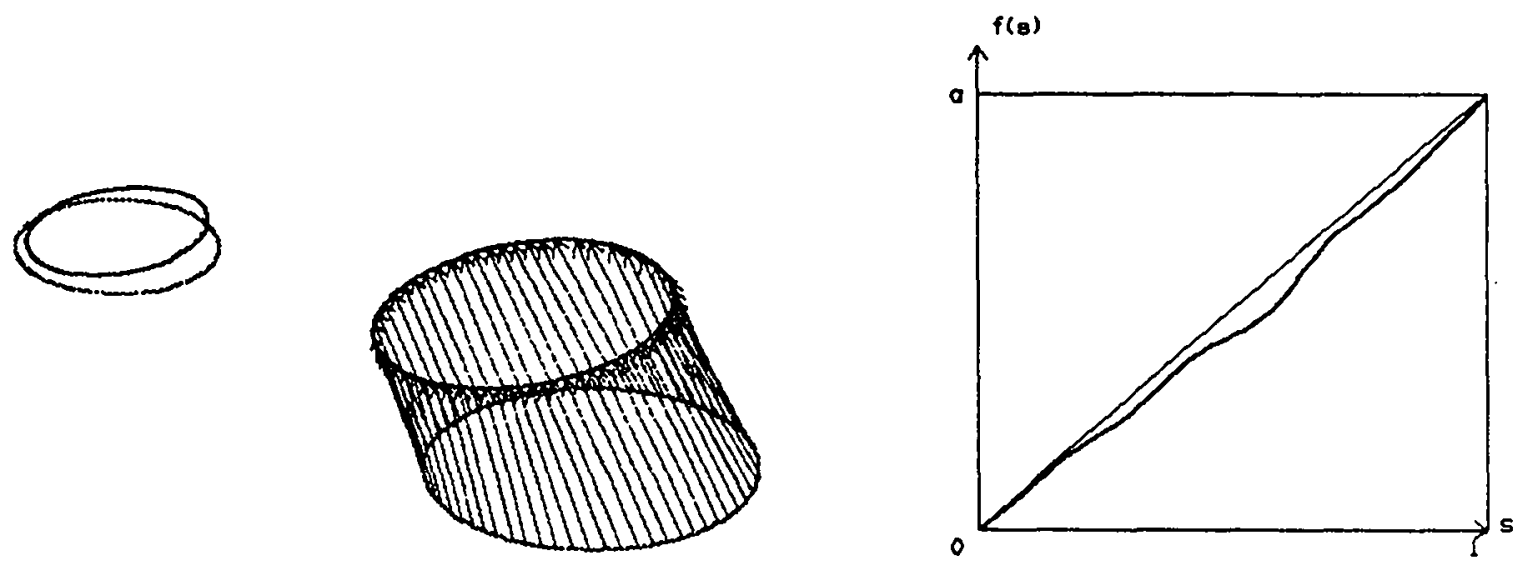

Figure 6: The optimal solution obtained by the algorithm. We remark that the each point of the black curve matches a single point of the grey curve, and that, maximum curvature points matched together. 

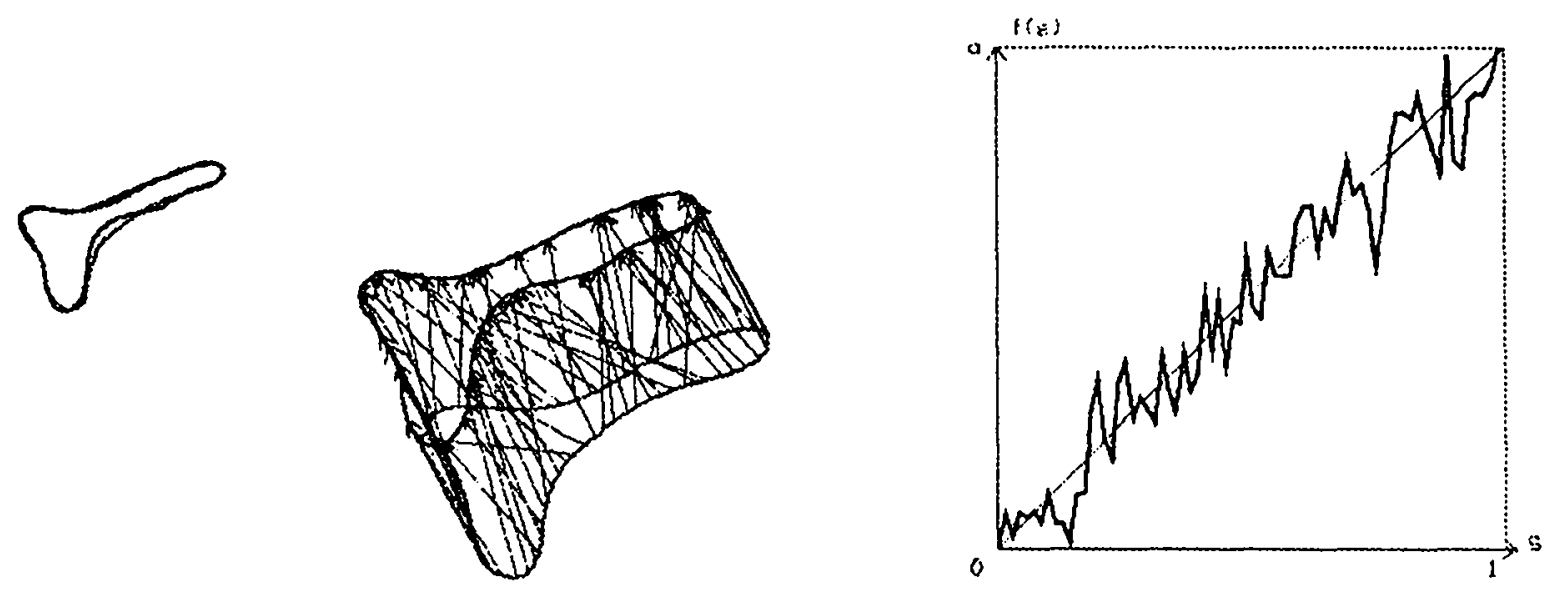

Figure 7: In this example we have corrupted an obtained solution with a gaussian noise $(\sigma=0.05)$ and considered this corrupted solution as an initial estimate $f_{0}$.
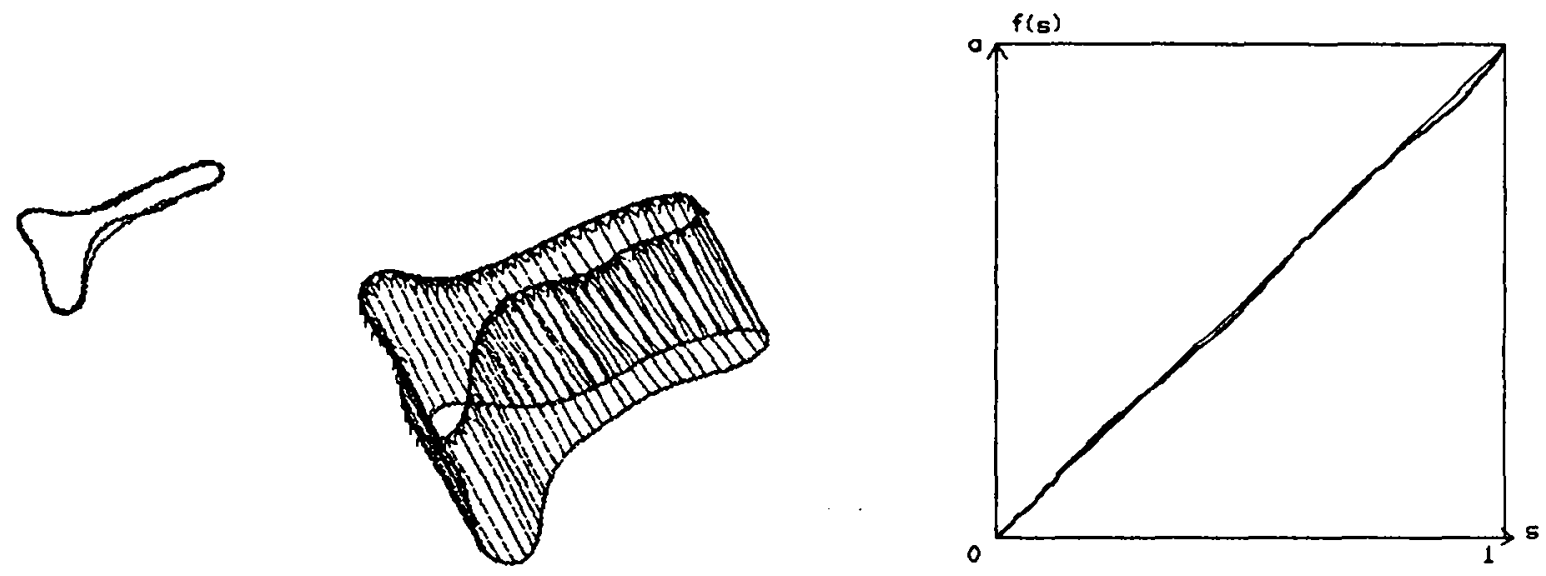

Figure 8: The minimum of $E$ starting with the initial estimation plotted in the previous figure. We have noted that if $\left\|f-f_{0}\right\|<4 h$ the algorithm still converges towards the realistic solution. 

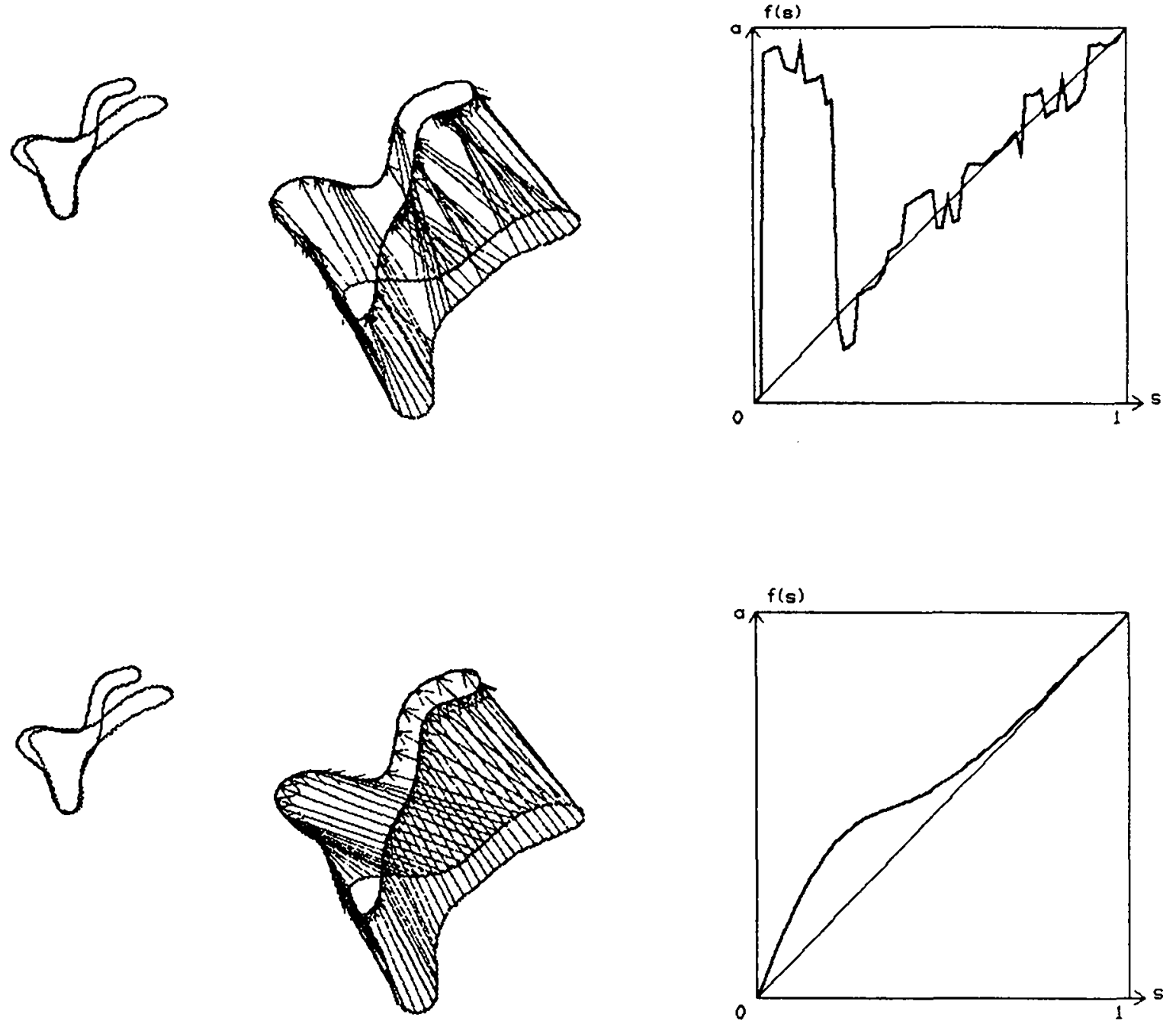

Figure 9: This example shows the problem that can occur in the computation of the initial estimate based only on the search in a given area. The initial estimation $f_{0}$ (top) and the obtained solution (bottom). 

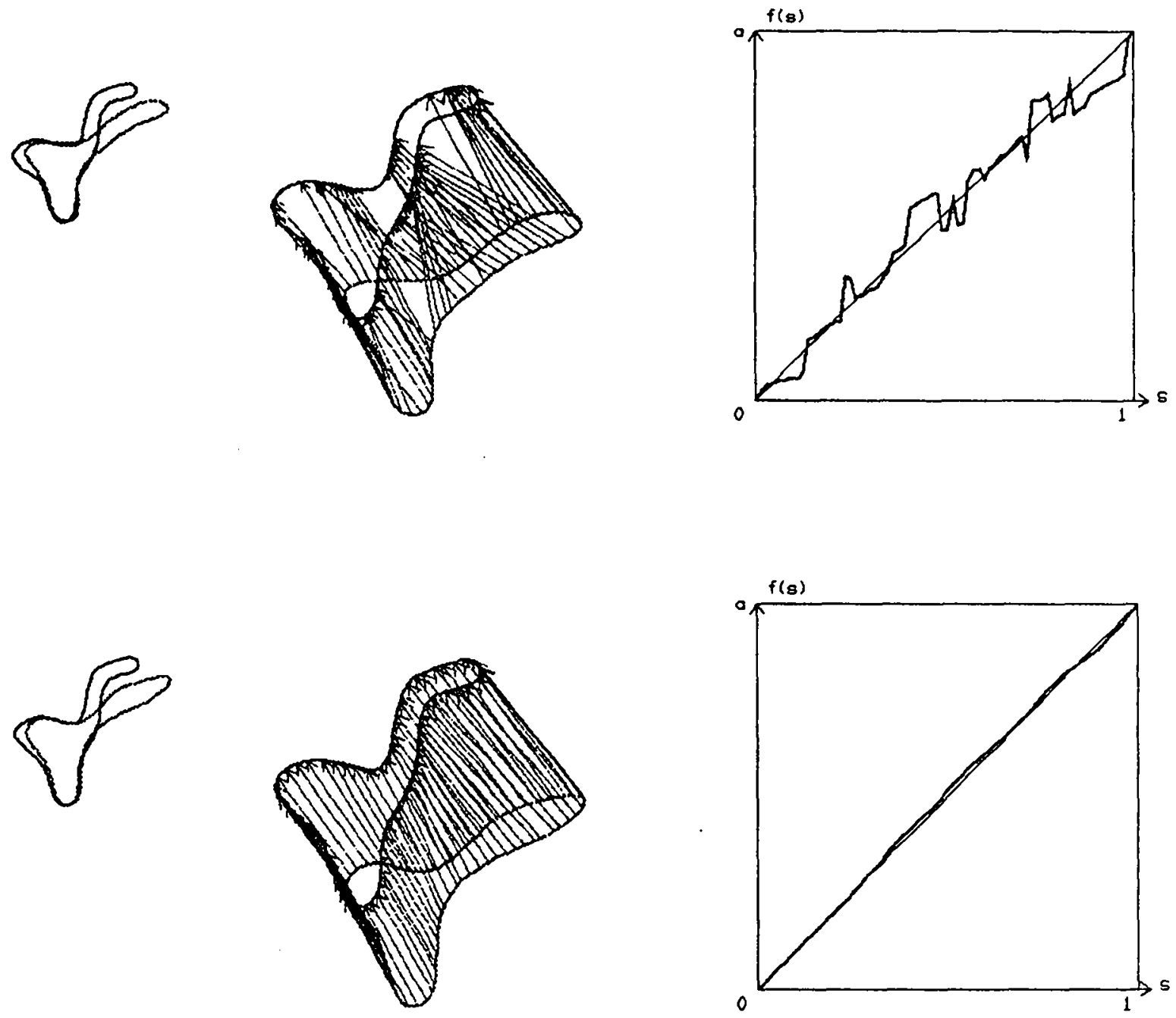

Figure 10: In the same case of the previous example, the computation of the initial estimate based on the local search and the curvilinear abscissa, gives a good estimation $f_{0}$, which leads to an acturate computation of the displacement function. 


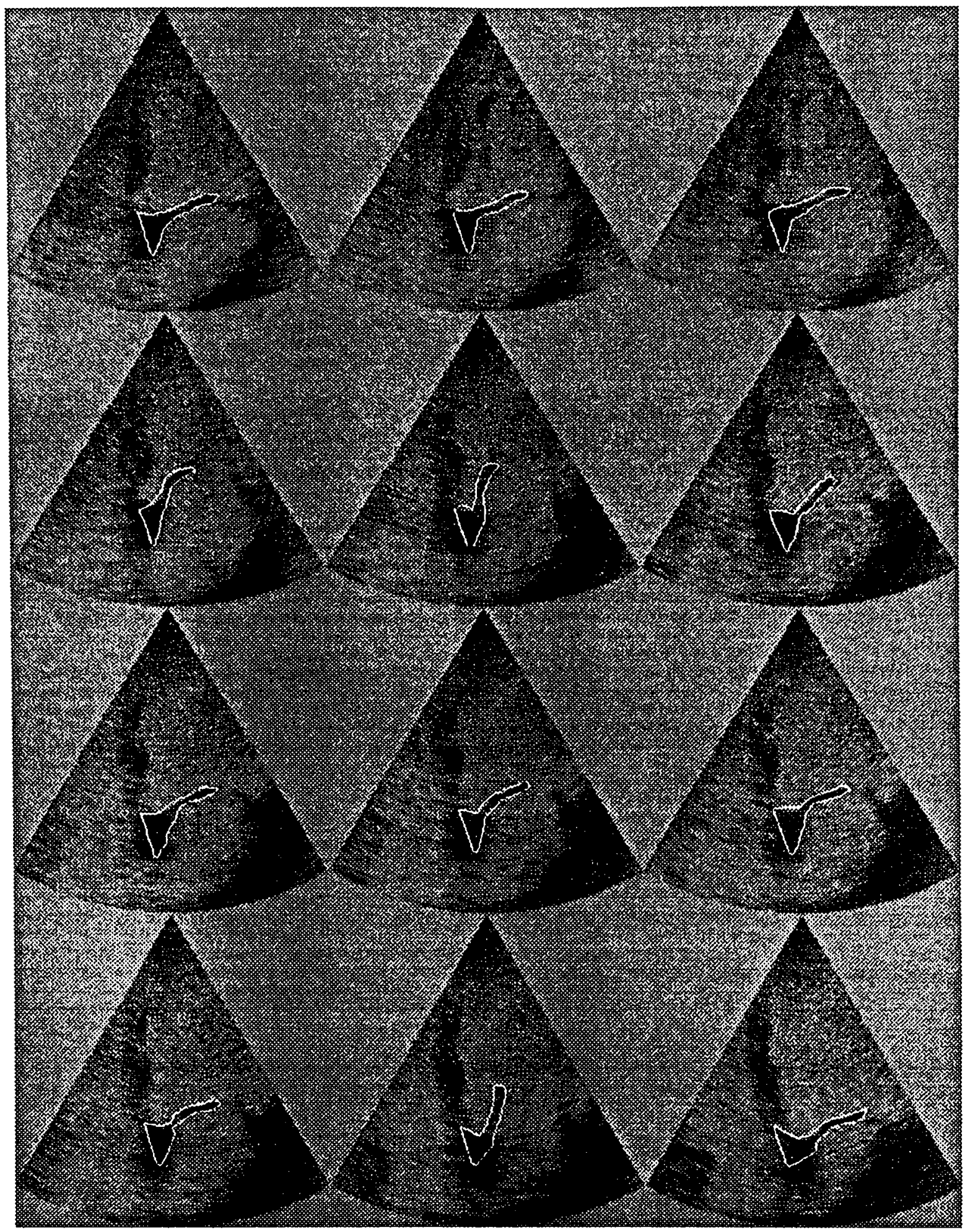

Figure 11: Temporal tracking of the mitral valve, obtained by the snake model[9]. 


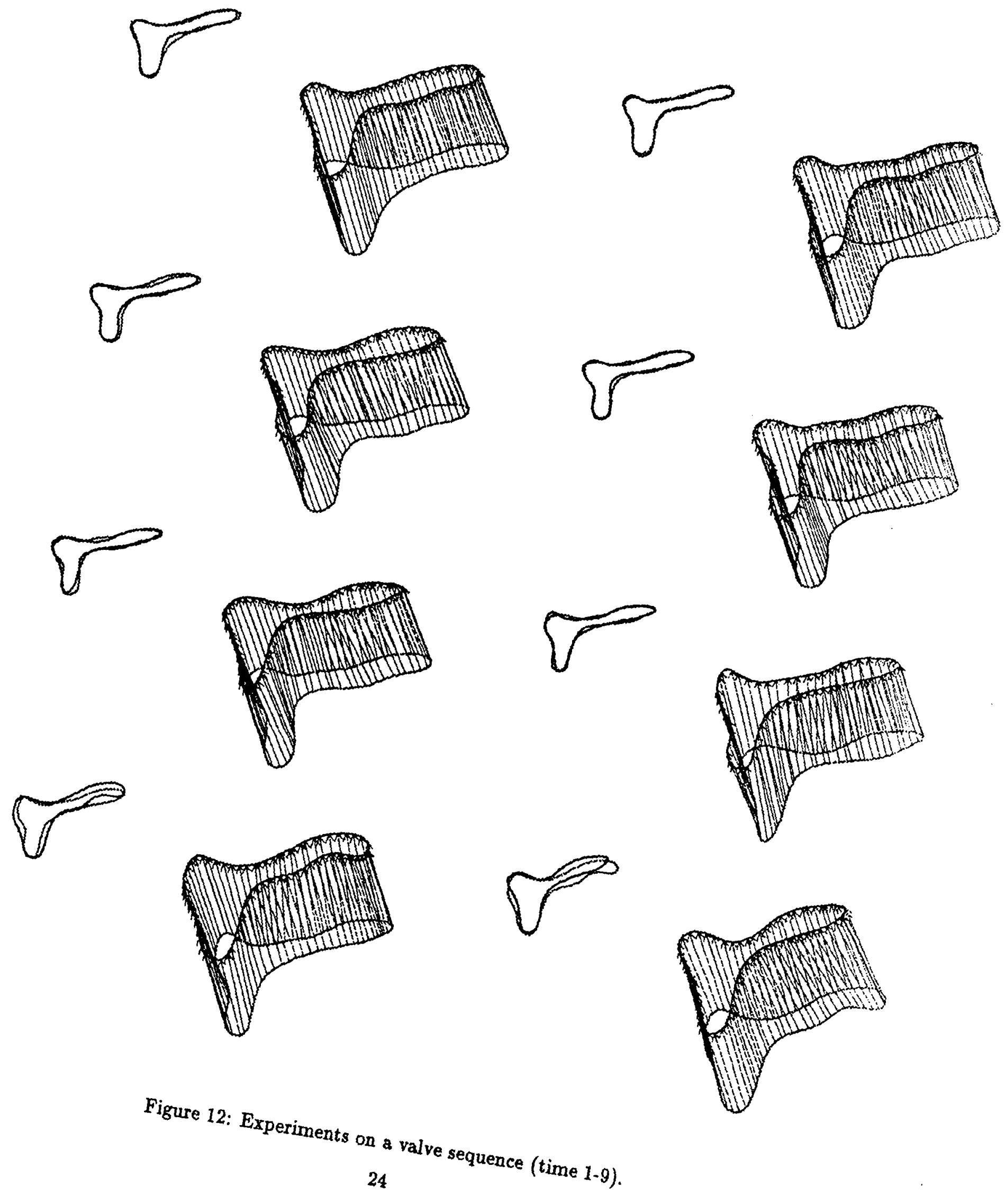



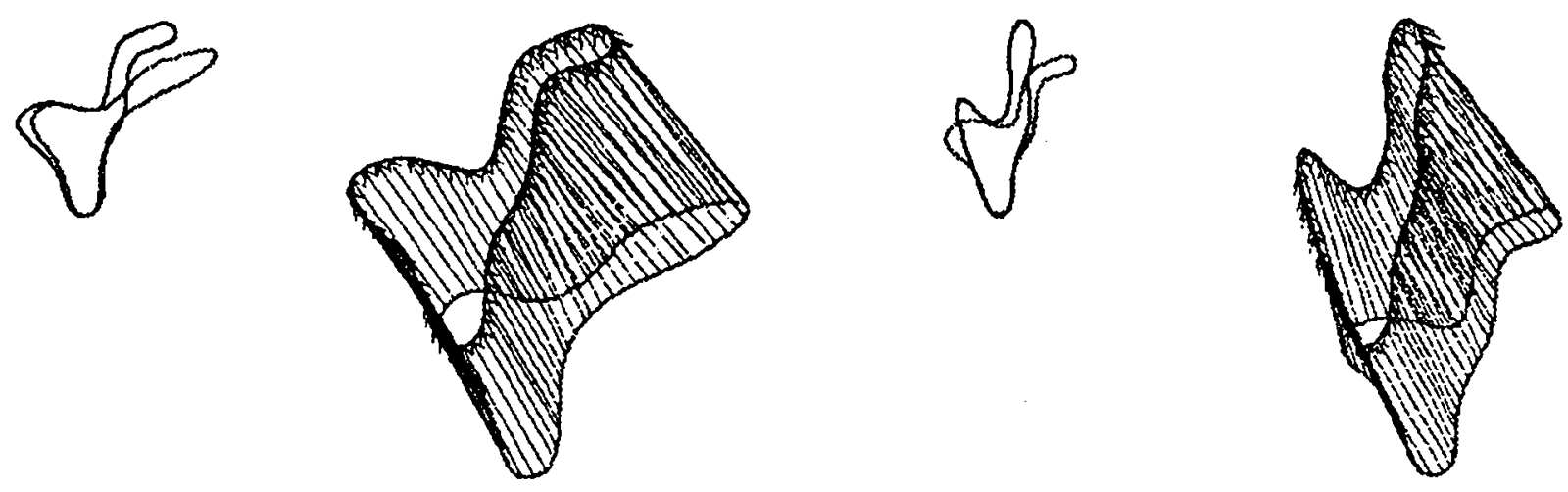

1
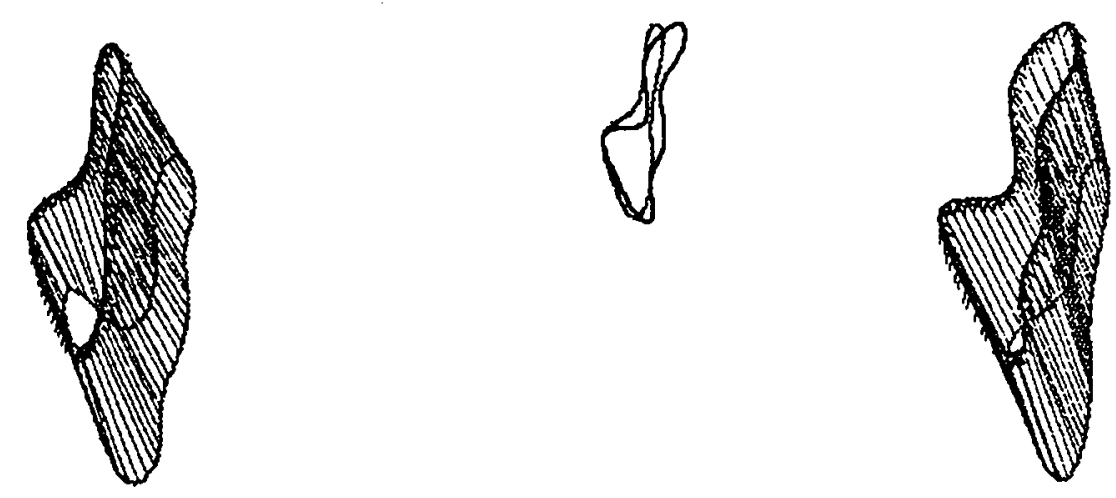

18
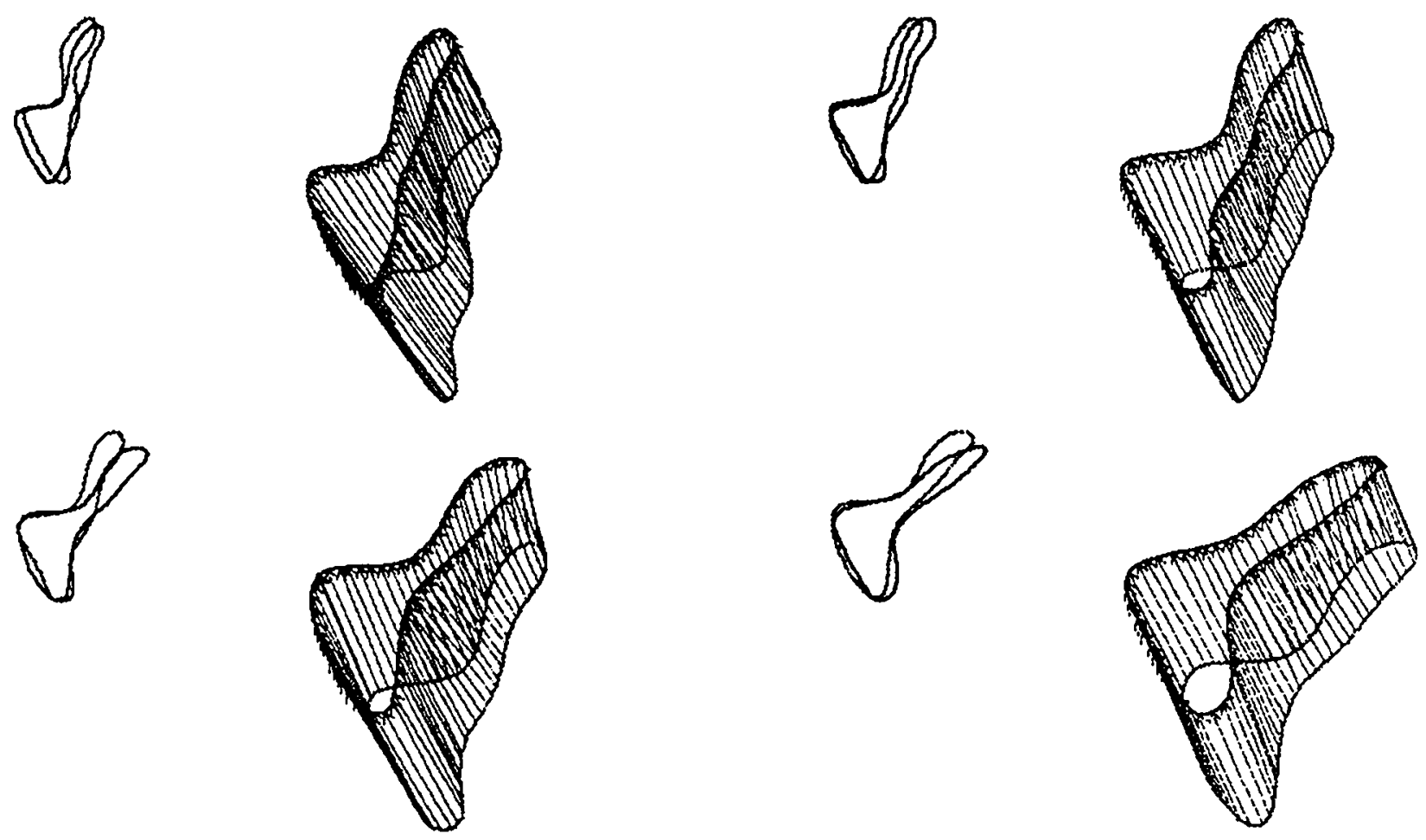

Figure 13: Experiments on a valve sequence (time 9-17) 

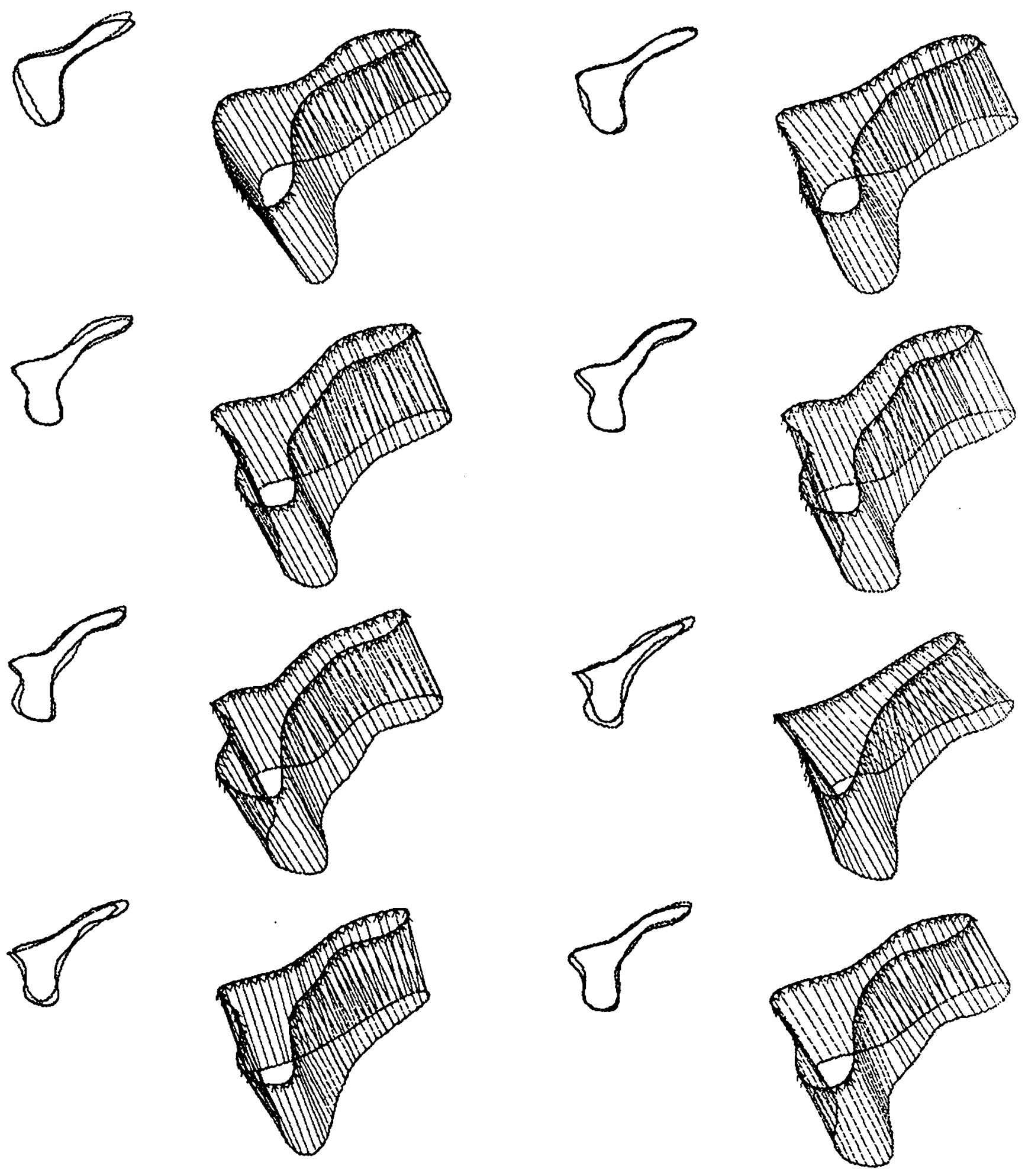

Figure 14: Experiments on a valve sequence (time 17-25) 

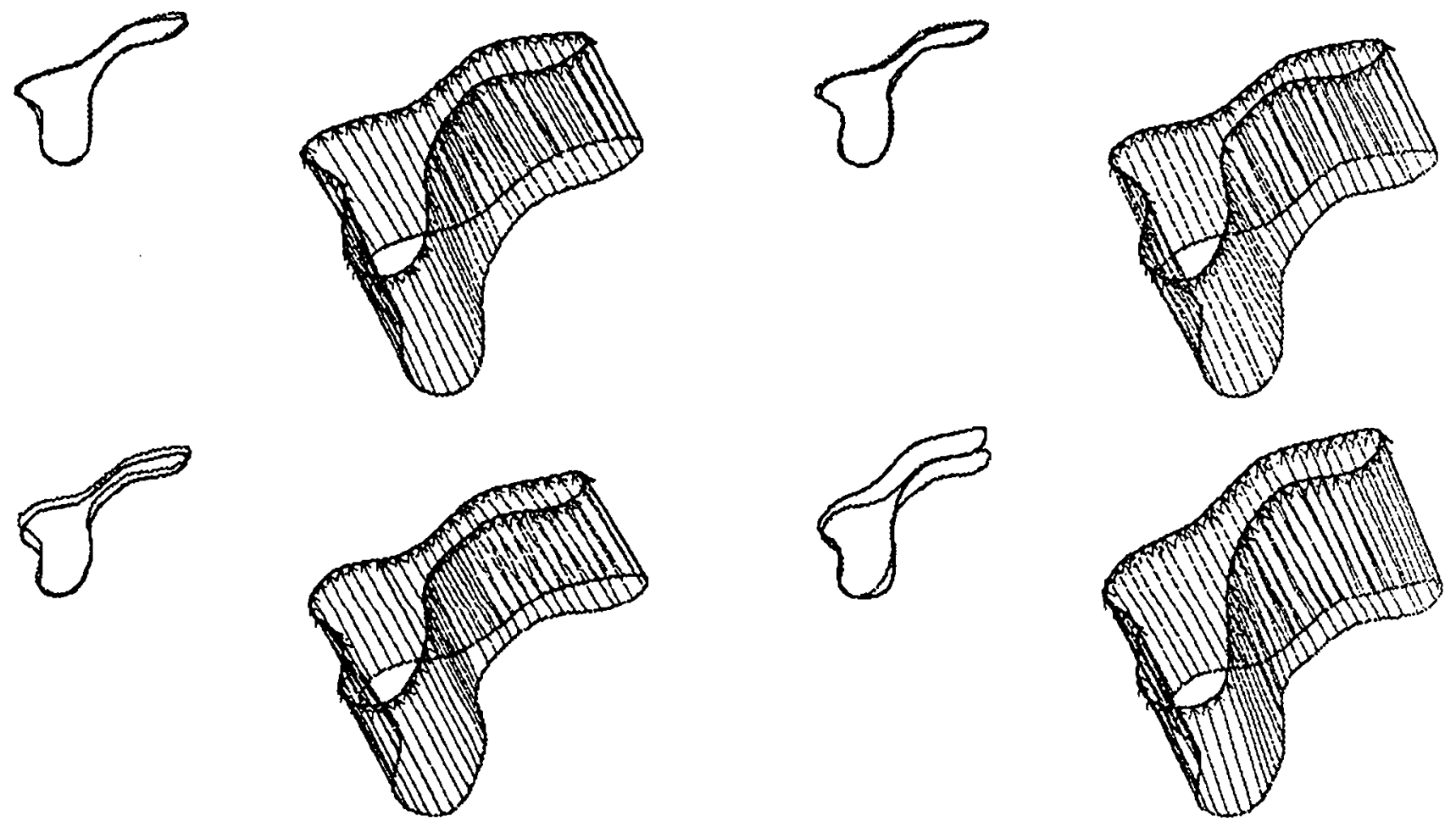

Figure 15: Experiments on a valve sequence (time 25-29) 
ISSN 0249-6399 